\title{
Implementation of Rigid Jacket for Flexible Nasofibroscopio 3D-printed in The Endoscopic Treatment of Subglottic Pathology: Presentation and Use in Clinical Cases
}

\author{
Juan Antonio Lugo Machado ${ }^{1 *}$, Edwin Canche Matin ${ }^{1}$ and Mario Sepúlveda ${ }^{2}$ \\ ${ }^{1}$ Medical Specialist in Otolaryngology, Instituto México del Seguro Social, Mexico \\ ${ }^{2}$ Instituto México del Seguro Social, Mexico
}

*Corresponding author: Juan Antonio Lugo Machado, Medical Specialist in Otolaryngology, Assigned to the otorhinolaryngology service Hospital de Especialidades No 2 "Lic. Luis Donaldo Colosio Murrieta" Centro Médico Nacional del Noroeste, Instituto México del Seguro Social, Cd. Obregón, Sonora, Mexixo.

To Cite This Article: Juan Antonio Lugo Machado. Implementation of Rigid Jacket for Flexible Nasofibroscopio 3D-printed in The Endoscopic Treatment of Subglottic Pathology: Presentation and Use in Clinical Cases. Am J Biomed Sci \& Res. 2019 - 2(3). AJBSR.MS.ID.000582. DOI: 10.34297/AJBSR.2019.02.000582

Received: March 23, 2019 | Published: April 08, 2019

\begin{abstract}
Summary
We present a surgical attachment that we call RIVEFLEXI3D for its acronym in English Rigid Vest for Flexible Nasolaryngoscope; no one similar or another that fulfills similar functions is registered. It has the objective of facilitating the reuse for therapeutic surgical purposes of the flexible endoscopy used in outpatient consultation. The instrument can be useful to optimize resources in hospitals with few material goods. It has been used in 4 patients operated on subglottic pathology, facilitating and optimizing the surgical treatment in these cases, without increasing costs or exposing patients to risks. We consider that its use should be extended to other hospital units that need to optimize material resources.
\end{abstract}

Keywords: Rigid Jacket for Flexible Nasofibroscope/instrumentation; Subglottis; Therapeutic laryngoscopy / 3D-printing

\section{Introduction}

The interest in seeing the larynx goes back to the beginning of the 19th century. The first pioneers used mirrors with the help of sunlight or a candle to see the larynx. Subsequently, Killian developed instruments for direct laryngoscopy with external lighting [1].

Surgical procedures in the larynx are part of the otolaryngologist's competence. The preferred surgical method for the treatment of laryngeal lesions under general anesthesia is microscopic endolaryngeal laryngoscopy (microlaryngosurgery) using a direct laryngoscope with a microscope [2].

The review of the literature on the endoscopic management of subglottic pathology implies sophisticated equipment and high costs, which some public hospitals in Mexico, Latin America and the world, do not count. We do not doubt that these teams are the ideal ones, however, the reality is very different in some countries like ours.

Direct laryngoscopy for the diagnosis and treatment of laryngeal diseases remains the gold standard, with direct vision under mi croscopy, adequate access to the supraglottis and glottis, however, the treatment of the pathology continues to be difficult. subglottic level and upper part of cervical trachea. Some authors point out a greater advantage with the use of rigid endoscopy compared to direct visualization with microscopy.

As Masahiro Kawaida and Griesdale in different studies indicate that video-assisted laryngoscopy provides an improved view of the larynx, compared to direct visualization with a microscope [3],[4].

\section{Justification for the Presentation of the Instrument}

Surgical procedures and diagnoses in airway are very common in medical practice and specifically in the otorhinolaryngological specialty, direct laryngoscopy with suspension laryngoscope and microscopy, it is still used as the standard technique to access the larynx, one of its limitations is that it suitably exposes the supraglottic and glottic region, but not the subglottic region and cervical trachea where there are entities, such as subglottic stenoses, hemangiomas, laryngeal papillomas with tracheal extension, granulomas of endolaryngeal tracheotomies, etc. 
Sophisticated equipment such as rigid $4 \mathrm{~mm}$ endoscopes of 0.30 and 70degrees, with the most suitable accessories to access these subglottic pathologies and cervical trachea, however in countries of low economic level, where this is not available, can be performed a 3D impression for the use of the flexible nasofibrocope used in the office and in this way simulate a rigid endoscope with the flexible fibroscope, giving it a dual function, both in the office and in the operating room.

\section{Material and Methods}

The standard surgical procedures of patients who underwent direct suspension microlaryngoscopy under balanced general anesthesia were performed, in 3 of the 4 patients they already had a tracheotomy prior to the procedure, they underwent a low tracheotomy (he retired after 72hours) with local anesthesia in a patient with recurrent respiratory papillomatosis with subglottic dissemination and cervical trachea to secure the airway.
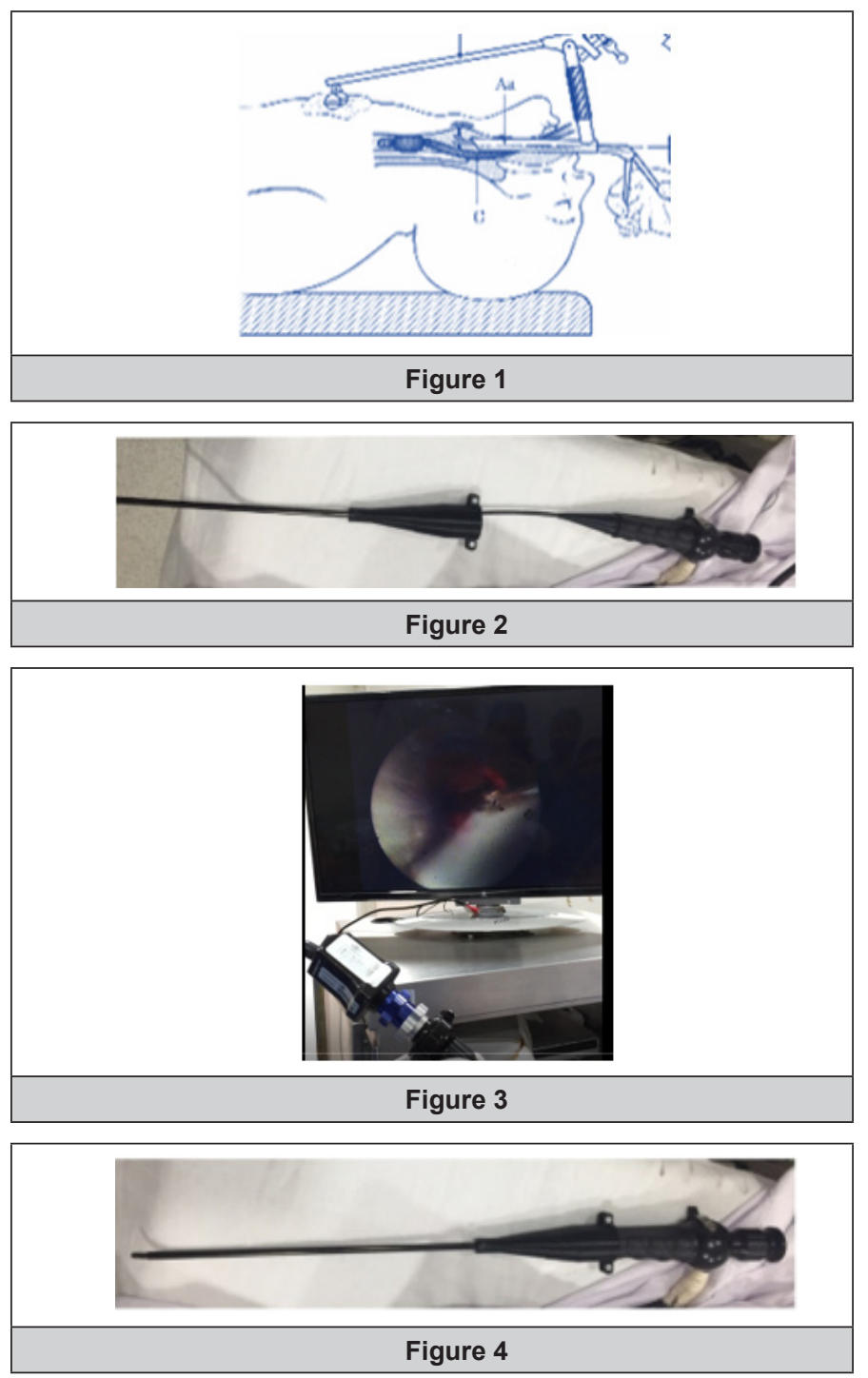

Under the anesthetic plan and with placement of the suspension laryngoscope (Figure 1) with the airway secured, the nasofibroscope is placed (Figure 2) with the camera and monitor (Figure 3), after which the rigid vest is installed printed in 3D (Riveflex I3D)
(Figure 4), to facilitate its introduction at the supraglottic, glottic, subglottic and cervical trachea level, where the pathology is located.

\section{Results and Discussion}

Four surgical procedures were performed in patients with subglottic involvement, facilitating access to that level with the new implement, reusing the nasofibroscope for surgical management in these cases, without raising costs or increasing risks to our patients. In case 1 , the tomography image of case 1 shows the cervical tracheal involvement due to invasion of the laryngeal papilloma, the subglottic and cervical trachea extension is observed, in the preoperative laryngoscopy image case 1 the extent of the disease is observed at the tracheal level, and in the laryngoscopy image case 1 of the immediate postoperative period, a permeable tracheal lumen is observed. In the following case, the case 2 preoperative laryngoscopy image with cotton-meyer II subglottic stenosis is observed, as well as the case 2 image of postoperative laryngoscopy an enlargement of the tracheal lumen is observed. In the preoperative laryngoscopy image of case 3 , the cotton-meyer II subglottic stenosis and the laryngoscopy image were observed 6 weeks after the dilation of case 3. Our last and fourth case is seen in the preoperative laryngoscopy image a subglottic stenosis cotton meyer IV, and in his postoperative laryngoscopy image of case 4 immediately his post-dilation result.

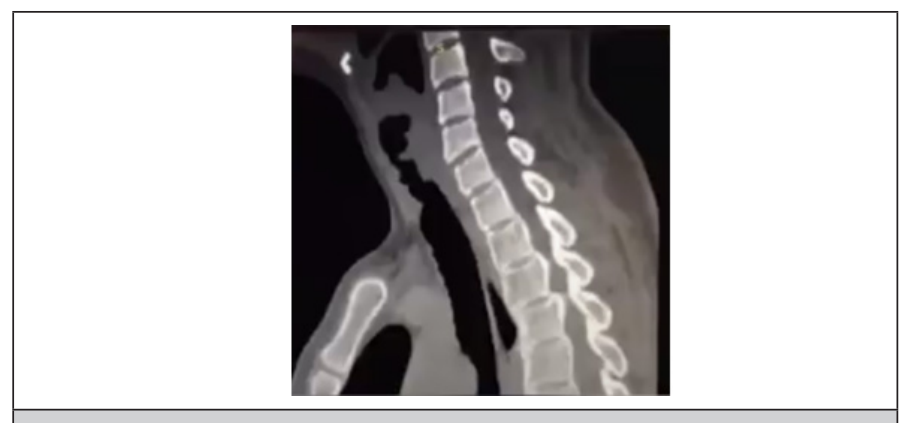

Figure 5a: Tomography image case 1.

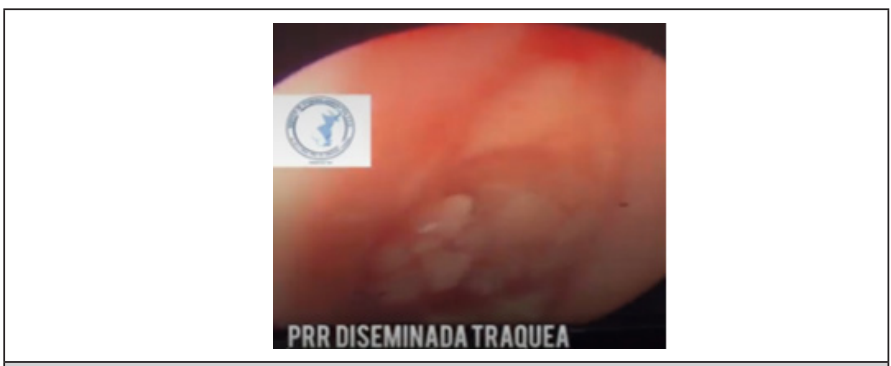

Figure 5b: Preoperative laryngoscopy image case 1.

Although we consider that the number of cases operated is small, we do understand that the results obtained so far are very satisfactory and that we will be carrying out a patient recruitment to give greater validity to our results and show us advantages and disadvantages of the addition according to a larger number of patients. cases we include. The present writing is for the purpose of publicizing the attachment and extending it to other places where material resources are scarce, and an office team can reuse for surgical purposes (Figure 5a-5i). 


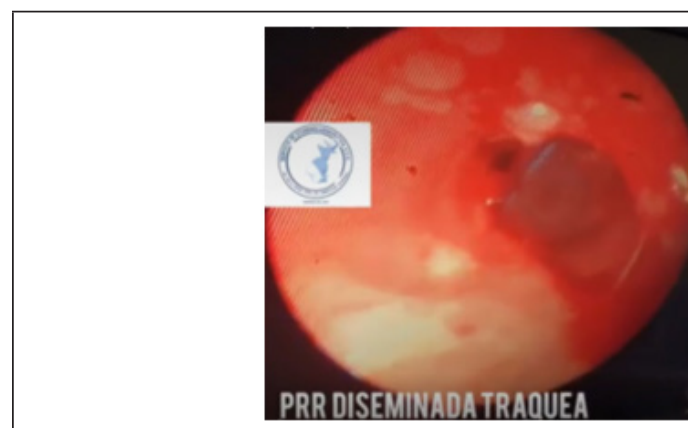

Figure 5c: Laryngoscopy image case 1 of the immediate postoperative period.

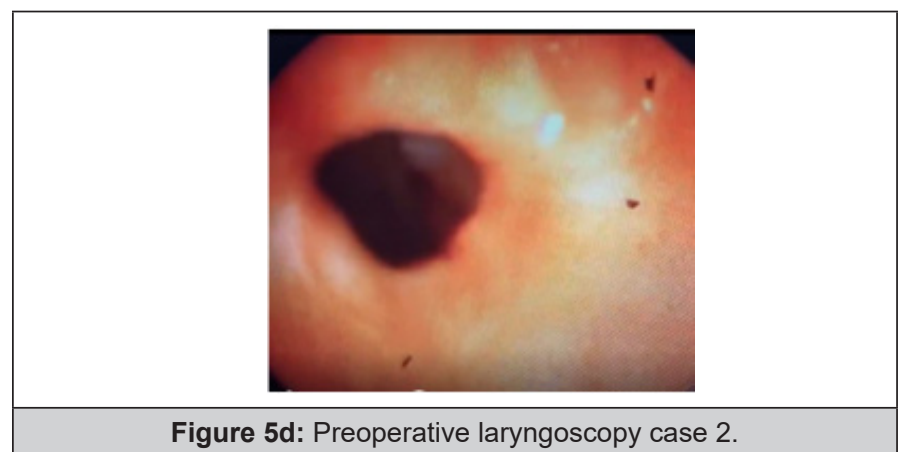

Figure 5d: Preoperative laryngoscopy case 2.

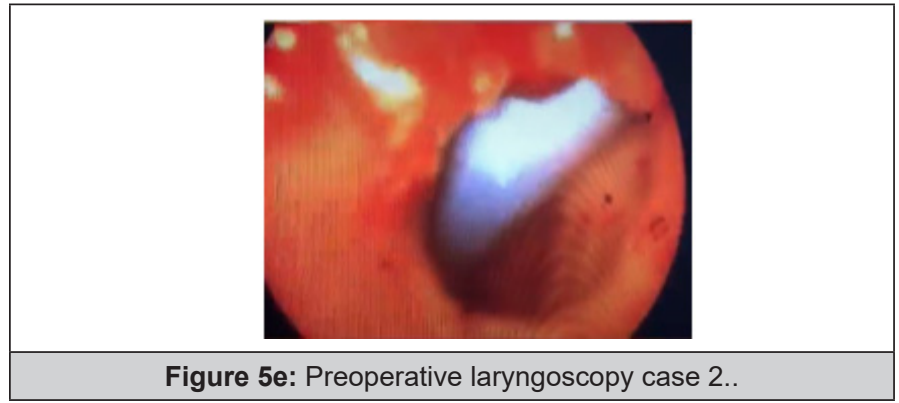

\begin{tabular}{|l|}
\hline ESG COTTON-MEYER II \\
DILATACIONNEUMATICA \\
\hline Figure 5f: Preoperative laryngoscopy case 3. \\
\hline
\end{tabular}

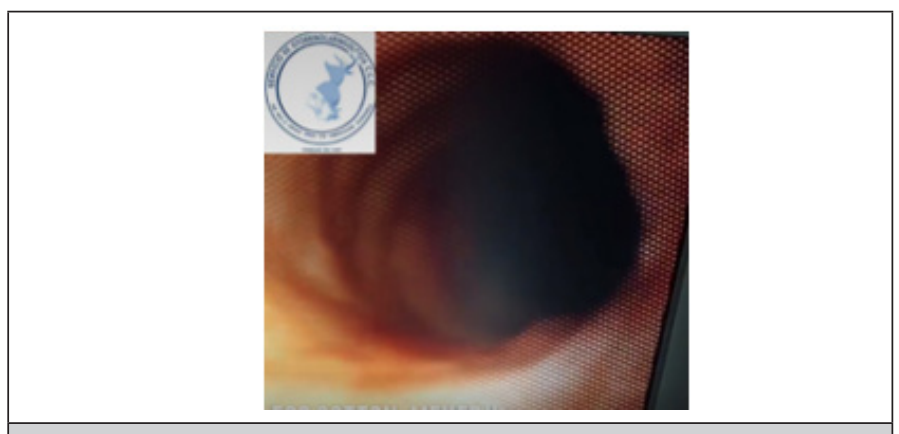

Figure 5g: laryngoscopy 6 weeks later case 3.

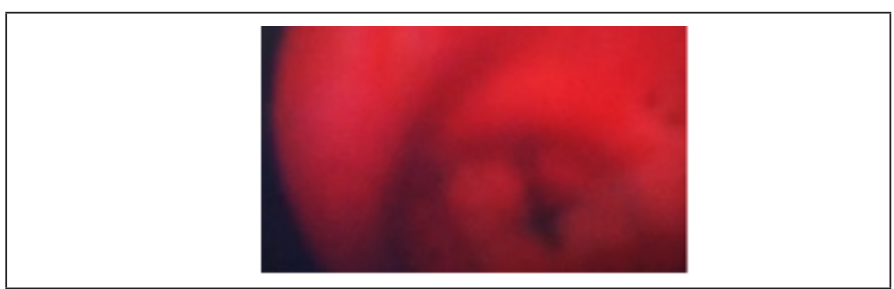

Figure 5h: Laryngoscopy stenosis subglotic cotton III clinical cases 4.

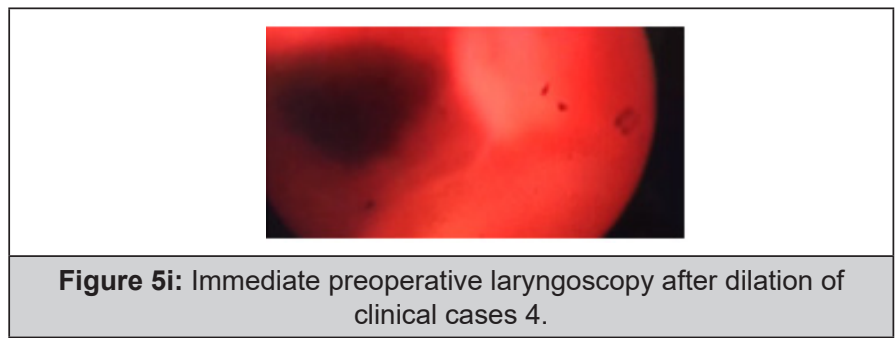

\section{References}

1. Mehta a, y chamyal, pc (2017) nuevo dispositivo de suspensión para procedimientos endoscópicos larargos. revista médica armed forces india, 50(4): 269-270.

2. Kawaida M, Fukuda H, y kohno, n (2000) laringoscopia endoscópica asistida por video utilizando un laringoscopio directo y un endoscopio largo y rígido. endoscopia diagnóstica y terapéutica 6(2): 51-7.

3. Masahiro Kawaida, Hiroyuki Fukuda, Naoyuki Kohno (2000) videoassisted endoscopic laryngosurgery using a direct laryngoscope and a long rigid endoscope. diagnostic and therapeutic endoscopy 6(2): 51-57.

4. Griesdale deg, Liu D, McKinney J, Choi PT (2012) glidescope® videolaryngoscopy versus direct laryngoscopy for endotracheal intubation: a systematic review and meta-analysis. can j anesth / j can anesth 59(1): 41-52. 